\title{
Three-Dimensional Analysis of Microbial Communities
}

\author{
B. Knierim ${ }^{1}$, B. van Leer ${ }^{2}$, R.I. Webb ${ }^{3}$, M. Lin ${ }^{1}$, T. Goddard ${ }^{4}$, P. Wilmes ${ }^{5}$, K. McDonald ${ }^{6}$, P. Hugenholtz ${ }^{7}$,
} J.T. Liphardt ${ }^{8}$, and M. Auer ${ }^{1}$

1. Lawrence Berkeley National Laboratory and Joint Bio-Energy Institute, 1 Cyclotron Road, Mailstop 001, Berkeley, CA 94720

2. FEI Company, 5350 NE Dawson Creek Dr., Hillsboro, OR 97124

3. Center for Microscopy and Microanalysis, University of Queensland, Brisbane, 4072, Australia

4. UCSF, Resource for Biocomputing, Visualization, and Informatics, 600 16th St., M/S 2240, San Francisco, CA 94158

5. Centre de Recherche Public - Gabriel Lippmann, 41 rue du Brill, L-4422 Belvaux, Luxemburg

6. Electron Microscope Lab, 26 Giannini Hall, UC Berkeley, Berkeley, CA 94720

7. Joint Genome Institute, 2800 Mitchell Drive, Walnut Creek, CA 94598

8. Physics Department, Stanley Hall 478, UC Berkeley, Berkeley, CA 94720

Biofilms are the predominant lifestyle for most microbes. Mixed microbial communities are adapted to their specific environment allowing them to live under sometimes extreme conditions. They display complex metabolic capabilities, such as the degradation of lignocellulosic biomass, presumably a consequence of their organization into complex communities, which often show high species interdependencies. We are particularly interested in the microbial communities that are capable of lignocellulose degradation. Our model system is the termite hindgut microbial community, arguably one of the most efficient lignocellulose degrading communities. While about 200 reported species reside in the hindgut [1] it is currently unknown how many of these are essential for the biological function of efficient lignocellulose degradation. Therefore this research is of great interest for the development of second and third generation biofuels. Our ultimate goal is to spatially map out the community members using correlative 16S rRNA Fluorescence In-Situ Hybridization (FISH) [2] and overlaying this information with TEM imaging. This technique allows for the identification of the bacteria and mapping of their macromolecular strategies, including the production of secreted vesicles or extracellularly located large macromolecular machines such as cellulosomes. Although being tedious, we can perform both techniques on the same thin section samples. Since imaging of thin sections only covers a small portion of the microbial communities and may be misleading due to arbitrary sectioning angle and position, we have employed Focused Ion Beam / Scanning Electron Microscopy (FIB/SEM) on high pressure frozen, freeze substituted and plastic embedded samples. This technique allows us to gain high-resolution insight (at 3.3x3.3x10 nm voxel size) into the three dimensional organization of the microbial community around the digested material. Unlike classical tomography, FIB/SEM allows imaging of fairly large volumes tens of microns in depth. Such complex and large data sets require sophisticated analysis and rendering. To this end, we have employed the software UCSF Chimera to visualize, segment and analyze the datasets. 
The data show a surprisingly organized structure of the bacterial cells towards the biomass they are digesting. We also find some unexpected results such as bacteria that have the complex digestion capabilities to form tunnel like structures all through the wood material, frequent intercellular connections and extracellular vesicles. [3]

\section{References}

[1] F. Warnecke et al., Nature 7169 (2007) 560.

[2] E. F. DeLong et al., Science 4896 (1989) 1360.

[3] This research was supported by the Director, Office of Science, of the US Department of Energy under contract DEAC03-76SF00098 and by a Feodor Lynen Research Fellowship from the Humboldt Foundation (to BK).

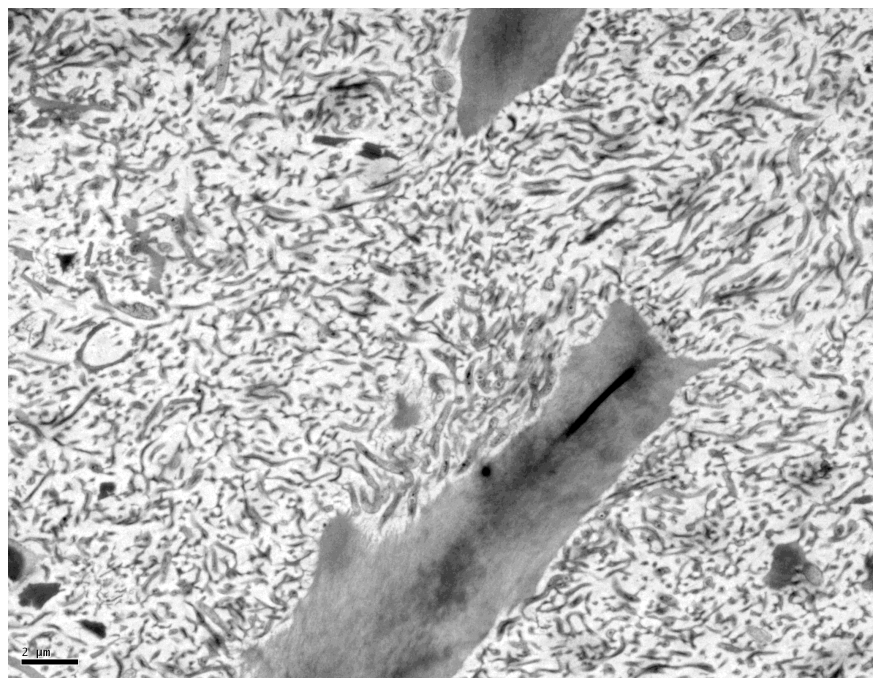

FIG. 1. TEM image of the termite hindgut microbial community. The bacteria fill all the space in the hindgut around the wood particles (large structures at the top and bottom).

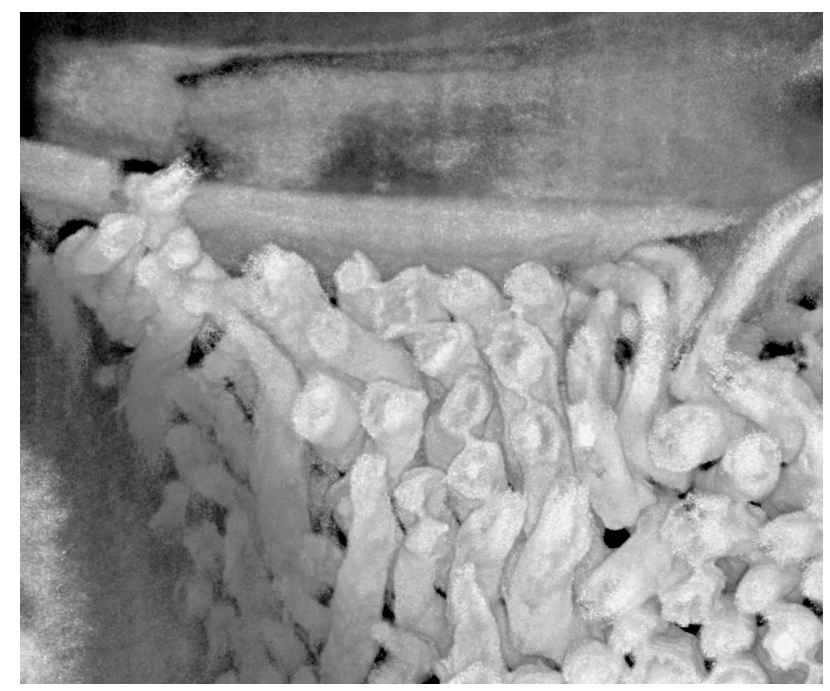

FIG. 2. Bacteria along a cell wall in one of the FIB/SEM datasets (acquired at FEI, Hillsboro). They show a parallel alignment towards the biomass. 